\title{
Nonstationary excitations in Bose-Einstein condensates under the action of periodically varying scattering length with time dependent frequencies
}

\author{
S. Rajendran, P. Muruganandam, and M. Lakshmanan* \\ Centre for Nonlinear Dynamics, Department of Physics, Bharathidasan \\ University, Tiruchirappalli - 620 024, India
}

\begin{abstract}
We investigate nonstationary excitations in 3D-Bose-Einstein condensates in a spherically symmetric trap potential under the modulation of scattering length with slowly varying frequencies (adiabatic modulation). By numerically solving the Gross-Pitaevskii equation we observe a step-wise increase in the amplitude of oscillation due to successive phase locking between driving frequency and nonlinear frequency. Such a nonstationary excitation has been shown to exist by an analytic approach using variational procedure and perturbation theory in the action-angle variables. By using a canonical perturbation theory, we have identified the successive resonance excitations whenever the driven frequency matches the nonlinear frequency or its subharmonics.
\end{abstract}

Key words:

Autoresonance, Bose-Einstein condensation, variational method, action-angle variable, canonical perturbation theory

PACS:

05.45-a, 46.40.Ff, 04.25.Nx, 03.75.Kk

* Corresponding Author. Fax:+91-431-2407093

Email address: lakshman@cnld.bdu.ac.in (M. Lakshmanan).

Preprint submitted to Elsevier 


\section{Introduction}

The successful experimental realization of trapped Bose-Einstein condensates in alkali metal atoms has triggered immense interest in understanding the various properties of the ultra cold matter [1,2]. The properties of the condensate wave function is usually described by a mean field Gross-Pitaevskii equation [3]. For the past couple of years, there has been increased interest in studying the properties of Bose-Einstein condensates with time varying trap potentials and scattering lengths both experimentally and theoretically [4,5,7,8,9]. In particular, temporal periodic modulation of scattering length by exploiting a Feshbach resonance is given a central importance in recent times. Earlier studies show that, in certain circumstances, periodically varying scattering length can stabilize the collapsing condensate $[6,7,8,9]$. However, very recently it has been shown that for a sign alternating nonlinearity an increase in the frequency of oscillations accelerates collapse [10].

From another point of view, these periodic modulations lead to resonance phenomena in the condensates. Resonance is an interesting feature of an oscillation under the action of an external periodic force manifesting in a large amplitude, when the frequency of the external force equals an integral multiple of the natural frequency of oscillation. Although, the phenomenon of resonance is well understood in linear systems, the nonlinearity that arises in the Bose-Einstein condensates due to the inter-atomic interactions leads to an important problem of nonlinear resonance of wide interest.

In general, there are two ways of driving a nonlinear oscillator by small driving periodic force, namely, external and parametric. If the driving frequency is constant (that is, driving force is exactly periodic with time), the initial growth of the oscillator's amplitude with time is arrested by its nonlinearity. On the other hand, if the driving frequency is slowly varying with time (driving force is almost periodic with time), the oscillator can stay phase locked but, on an average, increase its amplitude with time or a persistent growth in the amplitude takes place, and this phenomenon is known as autoresonance. In such systems, the nonlinear frequency is slowly varying with time. This autoresonance phenomena is also referred as adiabatic nonlinear phase locking and synchronization. However, in certain nonlinear systems, frequency is independent of the amplitude of oscillation [11,12]. In these systems, when the driving frequency is slowly varying with time, one might expect successive resonance excitations at subharmonic frequencies. We refer this type to resonance as a kind of subharmonic autoresonance [13].

In the present work, we identify such a subharmonic autoresonance in BoseEinstein condensates where it shows successive resonance excitations due to periodic modulation with slowly varying frequency. In Bose-Einstein conden- 
sates it is experimentally feasible to vary the scattering length by either magnetically or optically inducing a Feshbach resonance [4]14]. Earlier works on Bose-Einstein condensation report the periodic modulation with constant frequency of scattering length [5,7,8,9]. Along these lines, it is of potential interest to understand the dynamics of Bose-Einstein condensates under the action of periodically varying scattering length with slowly varying frequency. Motivated by the above, in this paper, we study the effect of such an excitation on the 3D Bose-Einstein condensates in a spherically symmetric trap potential and investigate the nature of parametric resonance. In particular, we point out the step-wise increase in amplitude of oscillation due to successive phase locking between the driving frequency and the nonlinear frequency (or its subharmonics) of the system.

This paper is organized as follows. In Sec. 2 we discuss the properties of Bose-Einstein condensates driven by a periodic force with time varying frequency from numerical simulations using pseudo-spectral method. In Sec. 3 we describe the variational procedure and derive a reduced system of ordinary differential equations (ODEs) to describe the dynamics of condensate width. In sec. 4 we analyze the width dynamics using a perturbed action angle variable theory for the reduced system of ODEs. Then in Sec. 5, by applying a canonical perturbation theory, we deduce the approximate nonlinear frequencies which are responsible for successive resonance excitation in BEC under the periodic modulation with slowly varying frequency. Finally in Sec. 6, we give a summary and conclusions.

\section{Nonlinear Gross-Pitaevskii Equation}

At ultra low temperatures, the time-dependent Bose-Einstein condensate wave function $\Psi(\mathbf{r} ; \tau)$ at position $\mathbf{r}$ and time $\tau$ may be described by the following mean-field nonlinear GP equation [3],

$$
\left[-\frac{\hbar^{2} \nabla^{2}}{2 m}+V(\mathbf{r})+\hat{g} N|\Psi(\mathbf{r} ; \tau)|^{2}-i \hbar \frac{\partial}{\partial \tau}\right] \Psi(\mathbf{r} ; \tau)=0 .
$$

Here $m$ is the mass and $N$ is the number of atoms in the condensate, $\hat{g}=$ $4 \pi \hbar^{2} \tilde{a} / m$ is the strength of interatomic interaction, with $\tilde{a}$ being the periodically varying atomic scattering length. The normalization condition of the wave function is $\int d \mathbf{r}|\Psi(\mathbf{r} ; \tau)|^{2}=1$. The three-dimensional trap potential is given by $V(\mathbf{r})=\frac{1}{2} m\left(\omega_{x}^{2} \bar{x}^{2}+\omega_{y}^{2} \bar{y}^{2}+\omega_{z}^{2} \bar{z}^{2}\right)$, where $\omega_{x}, \omega_{y}$, and $\omega_{z}$ are the angular frequencies in the $\bar{x}, \bar{y}$ and $\bar{z}$ directions, respectively, and $\mathbf{r} \equiv(\bar{x}, \bar{y}, \bar{z})$ is the radial vector (The standard notation $(x, y, z)$ is reserved below for rescaled variables). 


\subsection{Spherically symmetric trap potential}

In the spherically symmetric trap, i.e., $\omega_{x}=\omega_{y}=\omega_{z} \equiv \omega_{0}$ the trap potential is given by $V(\mathbf{r})=\frac{1}{2} m \omega_{0}^{2} \tilde{r}^{2}$, where $\omega_{0}$ is the angular frequency and $\tilde{r}$ the radial distance. The wave function can be written as $\Psi(\mathbf{r} ; \tau)=\psi(\tilde{r}, \tau)$. After a transformation of variables to dimensionless quantities defined by $r=\sqrt{2} \tilde{r} / l$, $t=\tau \omega_{0}, l \equiv \sqrt{\left(\hbar / m \omega_{0}\right)}$ and $\phi(r, t) \equiv \varphi(r, t) / r=\psi(\tilde{r}, \tau)\left(4 \pi l^{3}\right)^{1 / 2}$, the GP equation for the ground state wave function becomes

$$
\left[-\frac{\partial^{2}}{\partial r^{2}}+\frac{r^{2}}{4}+g\left|\frac{\varphi(r, t)}{r}\right|^{2}-i \frac{\partial}{\partial t}\right] \varphi(r, t)=0
$$

where $g=N \tilde{a} / l$. The normalization condition for the wave function is then

$$
\int_{0}^{\infty}|\varphi(r, t)|^{2} d r=2 \sqrt{2}
$$

\subsection{Numerical results}

In order to study the nature of the condensate wave function, we solve the GP equation (2) numerically using a pseudo-spectral method [15]. In the pseudospectral method the condensate wave function is expanded in terms interpolating polynomials. When this expansion is substituted into the GP equation, the (space) differential operators operate on a set of known polynomials and generate a differentiation matrix operating on the unknown coefficients. Consequently, the time-dependent partial-differential nonlinear GP equation in space and time variables is reduced to a set of coupled ordinary differential equations in time which can then be solved by a fourth-order adaptive step-size controlled Runge-Kutta method [16].

A similar pseudo-spectral method has been used in [17] with Hermite polynomials as the interpolant for the case of completely anisotropic trap potential. However, for the spherically symmetric condensates described by Eq. (2), it will be more advantageous to employ the integration in reduced dimensions with Laguerre polynomials as the interpolant. The Laguerre polynomials are more suited to the present problem as they are well defined in the interval $r \in[0, \infty)$ and satisfy the boundary conditions of the wave function of the GP equation [18].

We consider the case of periodically varying scattering length which can be

achieved by means of Feshbach resonance. In order to take into account the 
temporal modulation the scattering length can be taken as

$$
\tilde{a}(t)=\epsilon_{0}+\epsilon_{1} \cos \left[\int \omega(t) d t\right]
$$

and

$$
g(t)=\frac{N \tilde{a}(t)}{l}
$$

where

$$
\omega(t)=2 \omega_{0}+\delta-\mu t
$$

is the frequency of the applied field with $\mu \ll 1$ and $\delta$ is a constant. We choose the parameter, without loss of generality and within the critical threshold limit for collapse, $N=1$. The trap frequency $\omega_{0}$ is fixed at 1 . In order to study the resonance dynamics of (2), we calculate the root mean square distance, $\langle r\rangle=\sqrt{\left\langle r^{2}\right\rangle}$, which is defined as

$$
\left\langle r^{2}\right\rangle=\frac{1}{2 \sqrt{2}} \int_{0}^{\infty} r^{2}|\varphi(r, t)|^{2} d r
$$

When $\delta=0$ and $\mu=0$, the above system (2) exhibits nonlinear resonance in which the steady growth of the $\langle r\rangle$ is arrested by the nonlinear frequency of the system [17]. Fig. 11 shows the plot of $\langle r\rangle$ as a function of time obtained

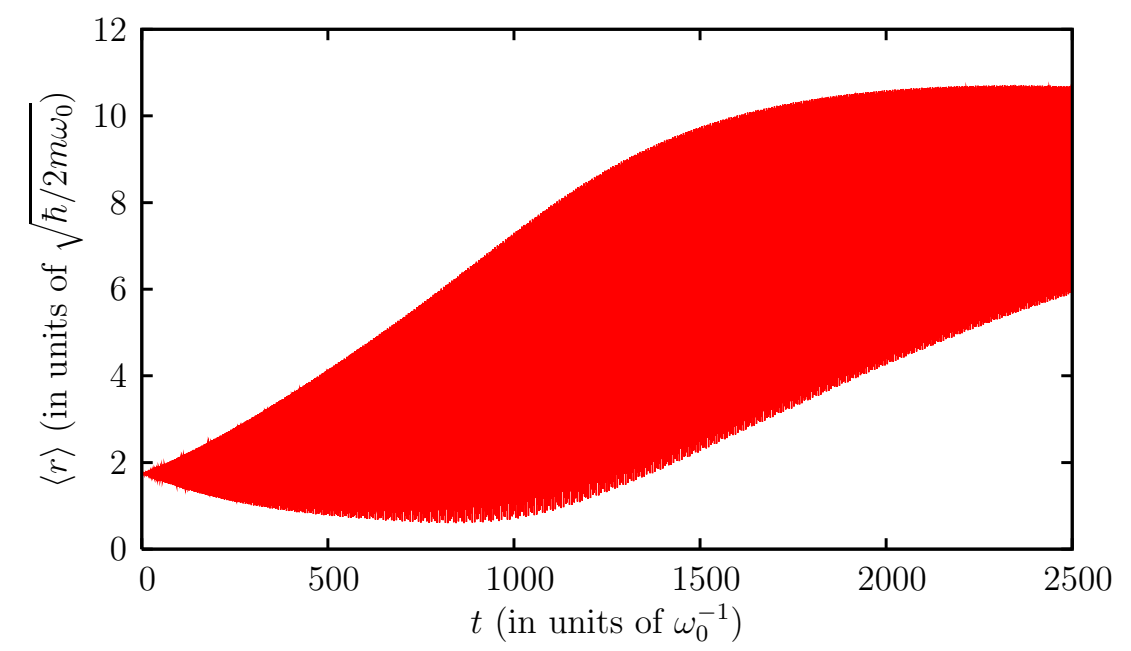

Fig. 1. Plot showing the $r m s$ value $\langle r\rangle$ as a function of time in the case constant frequency of the temporal modulation of the scattering length. The parameters are chosen as $\epsilon_{0}=0, \epsilon_{1}=0.01, \mu=0$ and $\delta=0$ in Eq. (4).

by solving (2) numerically for $\delta=0$ and $\mu=0$. Fig. 1 clearly indicates that the steady growth of $\langle r\rangle$ is controlled by the nonlinearity. However, for $\delta=0$ and $\mu=2 \times 10^{-3}$, one observes an interesting resonance phenomenon giving rise to a step-wise increase in $\langle r\rangle$ as illustrated in Fig. 2, To understand 


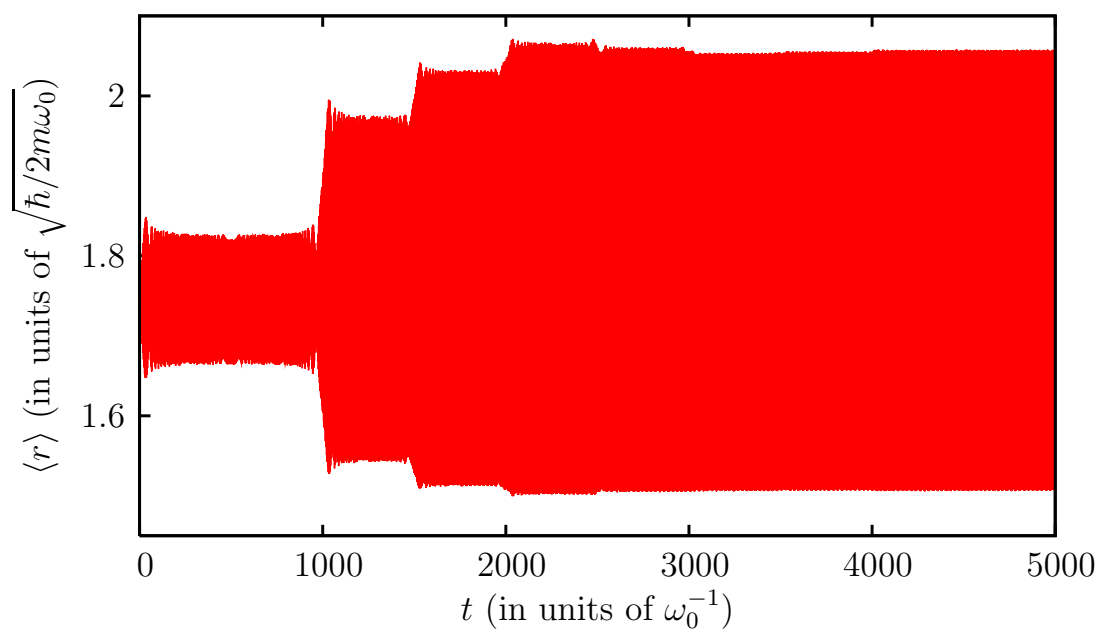

Fig. 2. Plot showing the $r m s$ value $\langle r\rangle$ as a function of time in the case slowly varying frequency of the temporal modulation of the scattering length. The parameters are chosen as $N=1, \omega_{0}=1, \epsilon_{0}=0, \epsilon_{1}=0.01, \mu=2 \times 10^{-3}$ and $\delta=0$ in Eq. (4).

such resonance excitations, we study the GP equation (2) by reducing it to a set of ODEs using a variational method and then by applying a canonical perturbation theory.

\section{Variational procedure}

The variational method is one of the simplest way to analyze the dynamics of Bose-Einstein condensate [7, 19,20]. In this method the original GP equation (2) is reduced to a system of ODEs, with fewer variables describing the condensate wave packet, by assuming suitable trial wavefunction [7,19,20. For convenience, Eq. (2) can be rewritten as (using the definition $\varphi(r, t)=r \phi(r, t)$ )

$$
i \frac{\partial \phi}{\partial t}=-\frac{\partial^{2} \phi}{\partial r^{2}}-\frac{2}{r} \frac{\partial \phi}{\partial r}+\frac{r^{2}}{4} \phi+g(t)|\phi|^{2} \phi
$$

According to the variational method we assume the Gaussian wave function in the form [7,8,9,19,20,21]

$$
\phi(r, t)=A(t) \exp \left[-\frac{r^{2}}{2 a(t)^{2}}+\frac{i b(t)^{2} r^{2}}{2}+i \delta(t)\right]
$$

where $A(t), a(t), b(t)$ and $\delta(t)$ are amplitude, width, chirp and phase, respectively [20], which are to be determined.

The equation for the wave packet parameters $A(t), a(t), b(t)$ and $\delta(t)$ can be 
obtained by calculating the average Lagrangian as [20]

$$
L(t)=4 \pi \int_{0}^{\infty} r^{2} \mathcal{L}(r, t) d r
$$

where

$$
\mathcal{L}(r, t)=\frac{i}{2}\left(\phi_{t}^{*} \phi-\phi_{t} \phi^{*}\right)+|\nabla \phi|^{2}+\frac{r^{2}}{4}|\phi|^{2}+\frac{g(t)}{2}|\phi|^{4}
$$

is the Lagrangian density.

One can easily see that the above Gaussian form assumption (17) leads to the Lagrangian density,

$$
\mathcal{L}(r, t)=\left[A^{2} r^{2}\left(\frac{\dot{b}}{2}+\frac{1}{a^{4}}+b^{2}+\frac{1}{4}\right)+A^{2} \dot{\delta}\right] e^{-r^{2} / a^{2}}+\frac{g(t)}{2} A^{4} e^{-2 r^{2} / a^{2}}
$$

and the average Lagrangian is given by

$$
L(t)=\frac{3 \pi^{3 / 2}}{2} A^{2} a^{5}\left(\frac{\dot{b}}{2}+\frac{1}{a^{4}}+b^{2}+\frac{1}{4}\right)+\pi^{3 / 2} A^{2} a^{3} \dot{\delta}+\frac{\pi^{3 / 2}}{8 \sqrt{2}} g(t) A^{4} \frac{a^{3}}{4} .
$$

The normalization condition for the wave function $\phi(r, t)$ as

$$
\int_{0}^{\infty} r^{2}|\phi(r, t)|^{2} d r=2 \sqrt{2}
$$

From the above condition we get $A^{2}=\frac{8 \sqrt{2}}{a^{3} \sqrt{\pi}}$. Therefore the average Lagrangian density can be rewritten as

$$
L(t)=\frac{16 \sqrt{2 \pi} g(t)}{a^{3}}+\frac{12 \sqrt{2} \pi}{a^{2}}+3 \sqrt{2} \pi a^{2}+12 \sqrt{2} \pi b^{2} a^{2}+6 \sqrt{2} \pi a^{2} \dot{b}+8 \sqrt{2} \pi \dot{\delta}
$$

Then the Euler-Lagrangian equations for the Lagrangian $L(t)$ lead to the following equations for the width $a(t)$ and chirp $b(t)$ :

$$
a_{t}=2 a b, \quad b_{t}=\frac{4 g(t)}{\sqrt{\pi} a^{5}}+\frac{2}{a^{4}}-2 b^{2}-\frac{1}{2} .
$$

On eliminating $b$ from the above Eq. (14), one is lead to the following evolution equation for $a$,

$$
a_{t t}+a=\frac{4}{a^{3}}+\frac{8 g(t)}{\sqrt{\pi} a^{4}} .
$$

The above equation (15) can be rewritten as

$$
a_{t t}+a=\frac{4}{a^{3}}+\frac{\epsilon(t)}{a^{4}}
$$


where

$$
\begin{aligned}
\epsilon(t) & =\frac{8 N}{\sqrt{\pi}}\left(\epsilon_{0}+\epsilon_{1} \cos \left[\int \omega(t) d t\right]\right), \\
\omega(t) & =2 \omega_{0}+\delta-\mu t .
\end{aligned}
$$

For $\epsilon(t)=0$, Eq. (16) is nothing but the well known Pinney equation [22]. This equation is completely solvable and the solution takes the following form 12

$$
a(t)=\left[A+\sqrt{A^{2}-4} \sin (2 t+\delta)\right]^{\frac{1}{2}} .
$$

Here $A$ and $\delta$ are arbitrary constants. The above nonlinear system Eq. (16) with $\epsilon(t)=0$ possesses the interesting dynamical property that the frequency of the system is completely independent of the amplitude, unlike the case of standard nonlinear oscillators. For exceptions, see ref. [11. However, for $\epsilon(t) \neq 0$ one has to study the full system Eq. (16) to understand the dynamics.

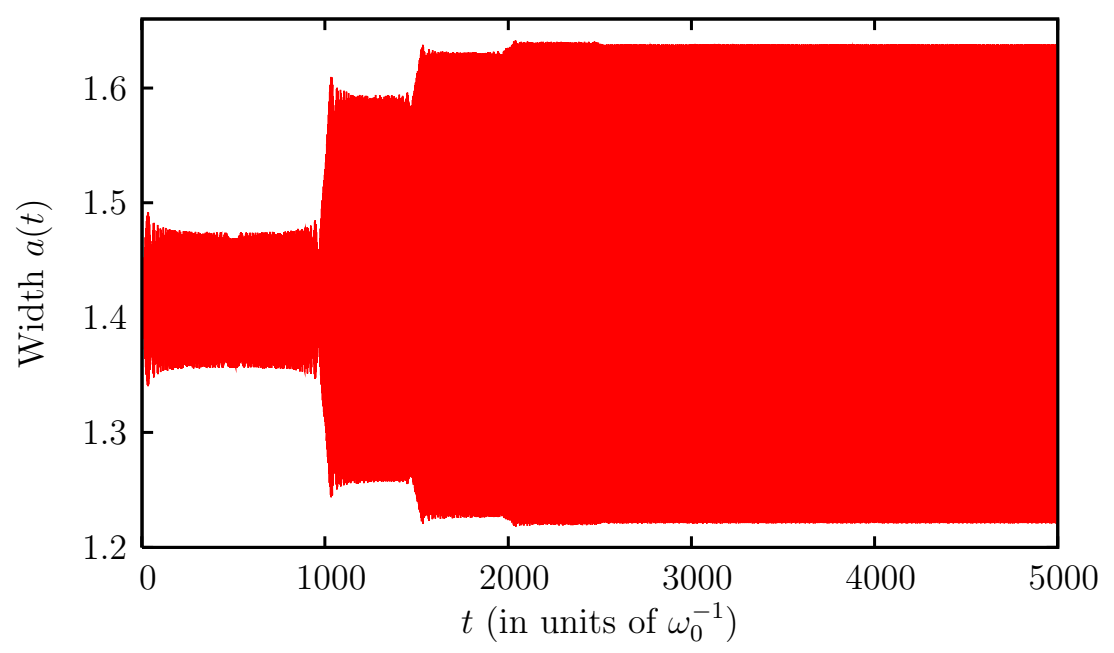

Fig. 3. Plot showing the width $a$ as a function of time calculated from equation (16). The parameters are $N=1, \omega_{0}=1, \epsilon_{0}=0, \epsilon_{1}=0.01, \mu=2 \times 10^{-3}$ and $\delta=0$.

In order to study the nature of oscillation for the width $a$ we solve the above equation (16) numerically using a fourth order Runge-Kutta method with appropriate initial conditions. For $\mu \neq 0(\mu=0.002)$, we observe that a stepwise increase in the amplitude of oscillation in $a$ as shown in Fig. 3. However, when $\mu=0$ this shows a usual nonlinear resonance phenomena [20].

One can easily relate the width $a(t)$ of the wavefunction obtaining from the the solution of the ODE (16) to the root mean square distance of the condensate 
$\langle r\rangle$ as

$$
\langle r\rangle=\left[\frac{1}{2 \sqrt{2}} \int_{0}^{\infty} r^{4}|\phi(r, t)|^{2} d r\right]^{\frac{1}{2}},
$$

which can be evaluated using the from of the wavefunction $\phi(r, t)$ [eq. (7)] and the fact that the amplitude of the wavefunction is given by the relation $A^{2}=\frac{8 \sqrt{2}}{a^{3} \sqrt{\pi}}$ obtained from the normalization condition (12). Thus the width of the condensate can be related to the root mean square distance of the condensate as $\langle r\rangle=a \sqrt{\frac{3}{2}}$.

\section{Perturbation theory in the action-angle variables}

In order to have a more clear understanding on the resonance phenomena, we analyze the Eq. (16) by constructing the action-angle variables.Treating Eq. (16) as nearly integrable system $(\epsilon(t) \ll 1)$, it is more convenient to consider the action-angle variables in which one can employ a perturbation theory [23].

\subsection{The action-angle variables for the unperturbed system}

First, let us consider the unperturbed system $\epsilon(t)=0$. The Hamiltonian for the unperturbed problem is given by

$$
H_{0}=\frac{a_{t}^{2}}{2}+U(a)
$$

where

$$
U(a)=\frac{a^{2}}{2}+\frac{2}{a^{2}}
$$

If $E$ is the energy then the width, $a(t)$, oscillates between $a_{\min }=\sqrt{E-\sqrt{E^{2}-4}}$ and $a_{\max }=\sqrt{E+\sqrt{E^{2}-4}}$. Since the Hamiltonian is conserved, i.e., $H_{0}=E$,

$$
\int \frac{d a}{\sqrt{2 E-a^{2}-4 a^{-2}}}=\int d t
$$

On integrating the above equation, we obtain

$$
a(t)=\sqrt{E+\sqrt{E^{2}-4} \sin \theta}
$$


where $\theta=\theta_{0}+2 t$ and $\theta_{0}$ being integration constant, see Eq. (19).

We now make a change to action-angle variables. In this problem, where the phase space is two-dimensional, there is one action variable $I$ and a conjugate angle variable $\theta$. The action variable is given by

$$
\begin{aligned}
I & =\frac{1}{2 \pi} \oint a_{t} d a \\
& =\frac{1}{\pi} \int_{a_{\min }}^{a \max } \sqrt{2 E-a^{2}-\frac{4}{a^{2}}} d a .
\end{aligned}
$$

This integral can be easily evaluated by using the energy $(E)$ to express the momentum $a_{t}$ in terms of position $a$. The resulting integral is elementary and leads to

$$
I=\frac{1}{2}(E-2)
$$

By expressing the total energy $E$ in terms of the action $I$, the unperturbed Hamiltonian is written as

$$
H_{0}=E=2(1+I)
$$

which is a function of $I$ only. The change of variables to action-angle variable is canonical, so that the Hamiltonian's equations retain their form

$$
\begin{aligned}
& \frac{d I}{d t}=-\frac{\partial H_{0}(I)}{\partial \theta}=0 \\
& \frac{d \theta}{d t}=\frac{\partial H_{0}(I)}{\partial I}=\Omega_{0}
\end{aligned}
$$

where $\Omega_{0}=2$.

\subsection{The action-angle variables for the system with perturbation}

Now we consider the problem with perturbation. Then the Hamiltonian for perturbed problem (16) can be written as

$$
H=H_{0}+\epsilon(t) H_{1} \equiv H_{0}+\epsilon(t) \frac{1}{3 a^{3}},
$$

where

$$
\epsilon(t)=\left(\frac{8 N \epsilon_{1}}{\sqrt{\pi}}\right) \cos \left(2 t+\delta t-\mu t^{2}\right), \quad \text { and } \epsilon_{0}=0
$$


Further, one can express $H$ in terms of action angle variables $I$ and $\theta$ using the Eqs. (22) and (25),

$$
H(I, \theta)=H_{0}(I)+\epsilon(t) H_{1}(I, \theta),
$$

where

$$
H_{1}(I, \theta)=\frac{1}{6 \sqrt{2}(1+I+\sqrt{I(2+I)} \sin \theta)^{3 / 2}},
$$

Thus the Hamiltonian equations of motion are given by
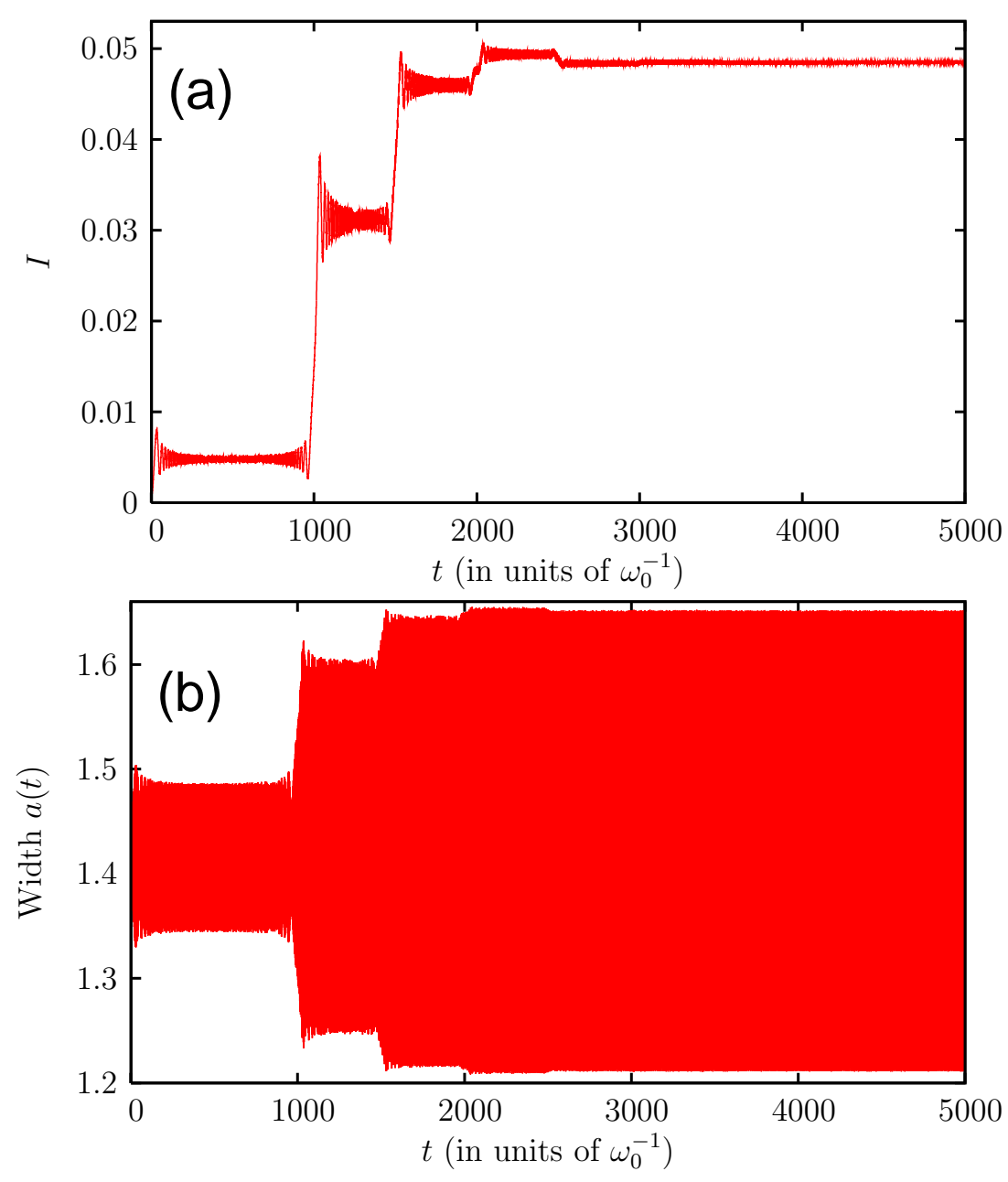

Fig. 4. Plot showing (a) the action $I$ as a function of time obtained by solving the reduced system Eq. (30) and (b) the width $a(t)$ obtained from eq. 31 as a function of $t$. The parameters are $N=1, \omega_{0}=1, \epsilon_{0}=0, \epsilon_{1}=0.01, \mu=2 \times 10^{-3}$ and $\delta=0$.

$$
\begin{aligned}
& \frac{d I}{d t}=-\epsilon(t) \frac{\partial H_{1}}{\partial \theta}, \\
& \frac{d \theta}{d t}=\Omega_{0}+\epsilon(t) \frac{\partial H_{1}}{\partial I}
\end{aligned}
$$


where

$$
\begin{aligned}
& \frac{\partial H_{1}}{\partial \theta}=-\frac{\sqrt{I(2+I)} \cos \theta}{4 \sqrt{2}(1+I+\sqrt{I(2+I)} \sin \theta)^{5 / 2}}, \\
& \frac{\partial H_{1}}{\partial I}=-\frac{1+\frac{1+I}{\sqrt{I(2+I)}} \sin \theta}{4 \sqrt{2}(1+I+\sqrt{I(2+I)} \sin \theta)^{5 / 2}} .
\end{aligned}
$$

The values of $I$ and $\theta$ are obtained, as a function of $t$, by solving Eqs. (30) numerically using a fourth order Runge-Kutta method with appropriate initial conditions. Fig. 4 (a) shows the plot of $I$ as a function of time. It is evident that $I$ retains relatively small values. Substituting $I$ and $\theta$ values in equations (25) and (22), we get the expression for condensate width $a$ in terms of action-angle variables as

$$
a=[2(1+I)+2 \sqrt{I(2+I)} \sin \theta]^{\frac{1}{2}}
$$

We note that the variation of $a$ [in Eq.(31)] depicted in Fig. 4(b), after using the results for $I$, compares well with that shown in Fig. 3. Further we have also

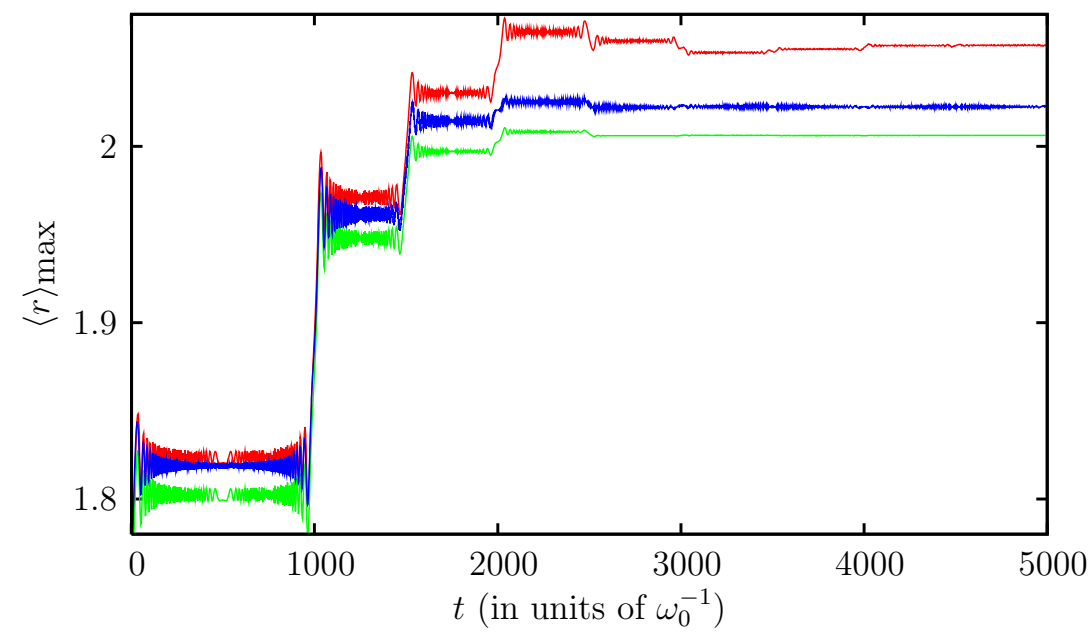

Fig. 5. Plot of the $\langle r\rangle_{\max }$ obtained by solving eq. (2), from the numerical solutions of eq. (15) and from perturbation theory (30.

compared, in Fig. 5, the results obtained from the direct numerical solution of the GP equation (2), using the numerical solution of (15) obtained from variational procedure and the results of the perturbation theory by solving equations (30). 


\section{Canonical perturbation Theory}

In order to understand the resonance phenomena one has to find out the approximate nonlinear frequency of the above system which is responsible for the resonant excitations. For this purpose we adopt a canonical perturbation theory to find the nonlinear frequency of near integrable systems [23,24|25]. The basic idea of this method is to rewrite the perturbed system (28) into a new set of action angle variables $(J, \phi)$ by a canonical transformation to a new Hamiltonian $K(J)$, which depends on the new action $J$ only, that is,

$$
H(I, \theta) \rightarrow K(J)
$$

For $I \ll 1$, one can express the perturbed Hamiltonian $H_{1}$ in the binomial expansion form as,

$$
H_{1}(I, \theta) \approx \frac{1}{6 \sqrt{2}}\left[1-\frac{3}{2}(I+\sqrt{I(2+I)} \sin \theta)\right]
$$

We now attempt a canonical transformation to action-angle variables $(J, \phi)$ for $H(I, \theta)$ through a type II generating function [26] as

$$
S(J, \theta)=S_{0}(J, \theta)+\epsilon(t) S_{1}(J, \theta)+\epsilon(t)^{2} S_{2}(J, \theta)+\ldots,
$$

where $S_{0}=J \theta$ is the identity generator. Then the new action-angle variables are given by 26 ]

$$
\begin{aligned}
& I=\frac{\partial S(J, \theta)}{\partial \theta}, \\
& \phi=\frac{\partial S(J, \theta)}{\partial J} .
\end{aligned}
$$

Rewriting the Hamiltonian (28) in terms of $J$ using the relation (34), such a transformation gives the new Hamiltonian $K(J)$, that is,

$$
H_{0}\left(\frac{\partial S(J, \theta)}{\partial \theta}\right)+\epsilon(t) H_{1}\left(\frac{\partial S(J, \theta)}{\partial \theta}, \theta\right)=K(J) .
$$

Furthermore, one now expands the new Hamiltonian $K(J)$ as a power series of $\epsilon(t)$, that is,

$$
K(J)=K_{0}(J)+\epsilon(t) K_{1}(J)+\epsilon(t)^{2} K_{2}(J)+\ldots
$$

By making a Taylor's series expansion of $H_{0}$ and $H_{1}$ in Eq. (35), using the relation for $S(J, \theta)$ from (33) , and equating the coefficients of various powers of $\epsilon(t)$, one can obtain a system of equations. The new Hamiltonian $K(J)$ can then be obtained by solving this set of equations. 
The zeroth order term $K_{0}(J)=H_{0}$ of the new Hamiltonian is obtained by equating the coefficient of $\epsilon(t)^{0}$. It is easy to see that $K_{0}(J)$ can be simply found by replacing $I$ by $J$ in the zeroth order Hamiltonian $H_{0}$. Therefore the zero-order frequency is given by,

$$
\Omega_{0}=\frac{\partial K_{0}}{\partial J}=2
$$

Similarly, equating the coefficient of $\epsilon(t)$, one obtains

$$
\begin{aligned}
K_{1}(J) & =\frac{\partial S_{1}}{\partial \theta} \frac{\partial H_{0}}{\partial J} H_{1}(J, \theta) \\
& =\Omega_{0} \frac{\partial S_{1}}{\partial \theta} H_{1}(J, \theta)
\end{aligned}
$$

At this point, one can exploit the periodicity of the motion in the angle variable $\theta$. Since $S_{i}, i=1,2, \ldots$, are assumed to be periodic in $\theta$. The averaging of Eq. (38) over $\theta$ leads to the mean of the derivatives of $S_{i}$ vanish. Then the first-order Hamiltonian correction is given by

$$
K_{1}(J)=1-\frac{3}{2} J
$$

Hence the first order correction to frequency is given by

$$
\begin{aligned}
\Omega_{1}(J) & =\frac{\partial K_{1}}{\partial J} \\
& =-\frac{3}{2} .
\end{aligned}
$$

from Eq. (34) upto first order,

$$
\begin{aligned}
& J=I-\epsilon(t) \frac{\partial S_{1}(I, \theta)}{\partial \theta}, \\
& \phi=\theta+\epsilon(t) \frac{\partial S_{1}(I, \theta)}{\partial \theta} .
\end{aligned}
$$

We get $I$ and $\theta$ values by solving Eq. (30) numerically and substituting these values in Eq. (41), we get the $J$ value.

The total nonlinear system frequency is,

$$
\Omega(J)=\Omega_{0}(J)+\epsilon(t) \Omega_{1}(J) .
$$

In the above expression the second term in the right hand side can be neglected as $\epsilon(t)$ is very small and one can find $\Omega(J) \approx \Omega_{0}(J)$. Here the nonlinear frequency is almost constant while the driving frequency is slowly varying with time. Hence one could expect a phase locking when the driving frequency matches with the nonlinear frequency or its integer multiples. 


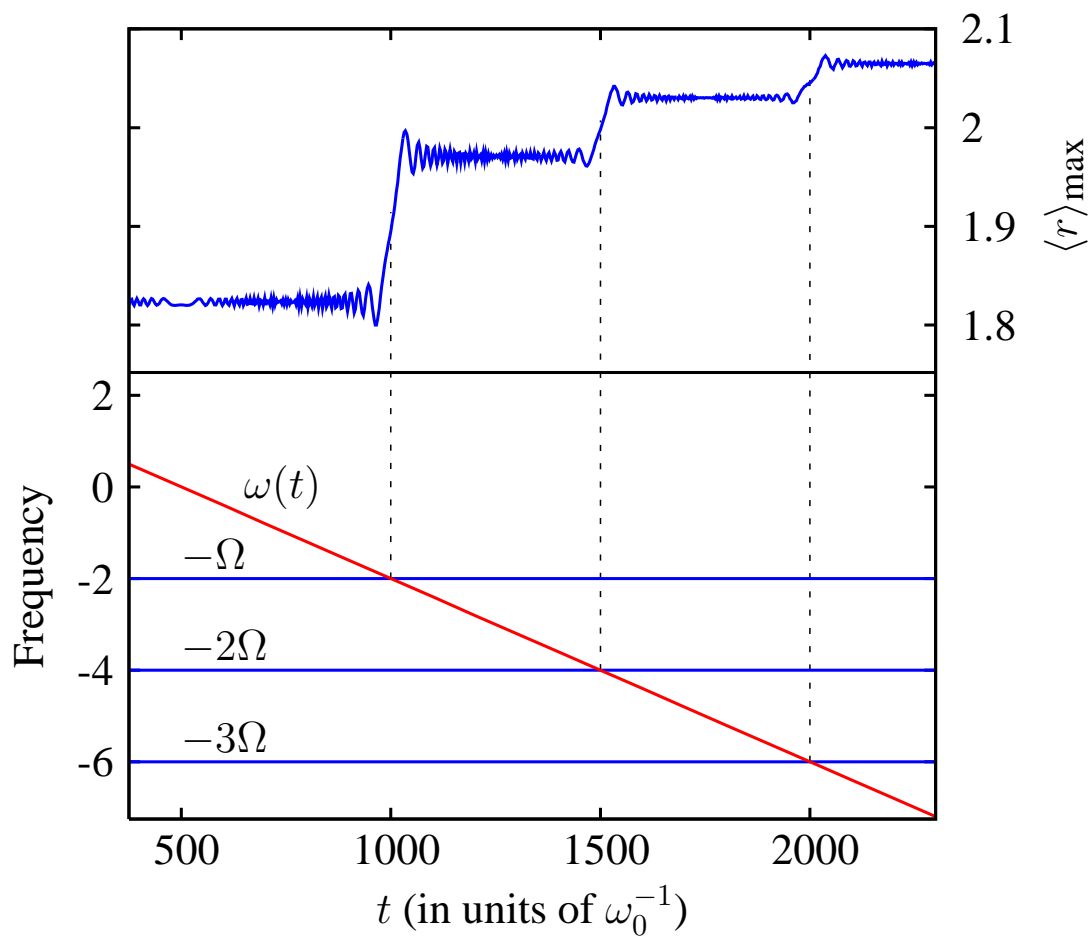

Fig. 6. Phase locking between nonlinear system frequency and driven frequency. (top) Plot of the $\langle r\rangle_{\max }$ obtained by solving Eq. (2) and (bottom) the driving frequency and the different harmonics of nonlinear frequency is shown.

From Fig. 6 it is easy to see that at $t \approx 1000$, the driving frequency $\omega(t)$ matches with the nonlinear system frequency $\Omega$ (i.e, $\omega(t)=-\Omega$ ), at which the first resonance jump occurs in the original problem as shown in Fig. 2 , It explains the primary resonance that occurs at $t \approx 1000$ in the original problem Eq.(2). At another time, $t \approx 1500$, where $\omega(t)=-2 \Omega$, a subharmonic resonance occurs leading to a second jump in the amplitude as shown in Fig. 2. As a result a step-wise increase in the amplitude as shown in Fig. 2 due to the phase locking between drive and system whenever the driving frequency matches with the system nonlinear frequency or its integral multiples (ie, $\omega(t)=n \Omega$, where $n=0, \pm 1, \pm 2, \ldots)$. We refer this phenomena as a kind of subharmonic autoresonance.

\section{Summary and conclusions}

In summary, we have studied the autoresonant excitations in Bose-Einstein condensates under the action of external periodic modulation with time dependent frequency. By numerically solving the corresponding Gross-Pitaevskii equation we have observed that there occurs a successive phase locking with step-wise increase in the overall amplitude of oscillation. We have employed a variational procedure using Gaussian trial wave function to simplify the 
problem in fewer coordinates. Then the reduced system has been found to show a kind of subharmonic autoresonance phenomenon with successive resonance excitations. Further, we have studied the problem in the action-angle variables which allows one to make a perturbation analysis and thereby identify the approximate frequency that is responsible for the successive resonant (step-wise) excitations. The results obtained from the canonical perturbation theory compare well with the numerical solution of the original problem.

\section{Acknowledgments}

This work is supported in part by the Department of Science and Technology (DST) and National Board for Higher Mathematics (Department of Atomic Energy) Government of India.

\section{References}

[1] K. B. Davis, M. O. Mewes, M. R. Andrews, D. S. Durfee, D. M. Kurn, W. Ketterle, Phys. Rev. Lett. 75 (1995) 3969.

[2] J. R. Enshera, D. S. Jin, M. R. Matthews, C. E. Wieman, E. A. Cornell, Phys. Rev. Lett.77 (1996) 4984.

[3] F. Dalfovo, S. Giorgini, L. P.Pitaevskii, S. Stringari, Rev. Mod. Phys.71 (1999) 463.

[4] S. Inouye, M. Andrews, J. Stenger, H. J. Miesner, D. Stamper-Kurn, W. Ketterle, Nature 392 (1998) 151.

[5] K. Staliunas, S. Longhi, G. J. de Valcácel, Phys. Rev. Lett. 89 (2002) 210406.

[6] I. Towers and B. A. Malomed J. Opt. Soc. Am. B 19 (2002) 537.

[7] H. Saito, M. Ueda, Phys. Rev. Lett. 90 (2003) 040403.

[8] F. K. Abdullaev, J. G. Caputo, R. A. Kraenkel, B. A. Malomed, Phys. Rev. A 67 (2003) 013605.

[9] S. K. Adhikari, Phys. Rev. A 69 (2004) 063613.

[10] V. V. Konotop, P. Pacciani, Phys. Rev. Lett. 94 (2005) 240405.

[11] V. K. Chandrasekar, M. Senthilvelan, M. Lakshmanan, Phys. Rev. E 72 (2005) 066203.

[12] V. K. Chandrasekar, M. Senthilvelan, M. Lakshmanan, in: Proc. 3rd Natnl. Conf. Nonlinear Systems \& Dynamics, Univ. Madras, Chennai, 2006, pp. 3-6. 
[13] L. Friedland, Phys. Rev. E 61 (2000) 3732.

[14] F. K. Fatemi, K. M. Jones, P. D. Lett, Phys. Rev. Lett. 85 (2000) 4462.

[15] P. Muruganandam, S. K. Adhikari, in: Proc. First Natnl. Conf, Nonlinear Systems \& Dynamics, IIT, Kharaghpur, 2003, pp. 76-79.

[16] W. H. Press, B. P. Flannery, S. A. Teukolsky, W. T. Vetterling, Numerical Recipes in Fortran 77: The Art of Scientific Computing, Cambridge University Press, Cambridge, 1993.

[17] P. Muruganandam, S. K. Adhikari, J. Phys. B: At. Mol. Opt. Phys. 36 (2003) 2501.

[18] M. Edwards, K. Burnett, Phys. Rev. A 51 (1995) 1382.

[19] D. Anderson, Phys. Rev. A 27 (1983) 3135.

[20] F. K. Abdullaev, J. C.Bronski, R. M. Galimzyanov, Physica D 184 (2003) 319.

[21] S. K. Adhikari, Phys. Rev. E 70 (2004) 036608.

[22] E. Pinney, Proc. Amer. Math. Soc. 1 (1950) 681.

[23] A. J. Lichtenberg, M. A. Lieberman, Regular and Stochastic Motion, Springerverlag, New York, 1983.

[24] M. Tabor, Chaos and Integrability in Nonlinear Dynamics, John Wiley \& Sons Inc., New York, 1989.

[25] M. Lakshmanan, S. Rajasekar, Nonlinear Dynamics: Integrability, Chaos and Patterns, Springer-verlag, Berlin, 2003.

[26] H. Goldstein, Classical Mechanics, Addission-Wesley, Reading, 1980. 